\title{
Loran-C Skywave Delay Detection Using the ARMA Algorithm
}

\author{
Abbas Mohammed \\ Department of Applied Signal Processing, \\ University of Karlskrona/Ronneby, \\ S-372 25 Ronneby, Sweden. \\ David Last \\ School of Electronic Engineering and Computer Systems, \\ University of Wales, Bangor, Gwynedd LL57 1UT, UK.
}

\begin{abstract}
The autoregressive moving average technique (ARMA) is applied to the problem of estimating the delay of Loran-C skywaves. The performance of this technique is evaluated and compared with Fourier-based methods. The simulation results show that the ARMA algorithm yields significantly greater estimation accuracy and that it can also operate successfully with very noisy signals.
\end{abstract}

\section{Introduction}

A Loran-C receiver calculates its position by measuring the time of arrival (TOA) of groundwave signals from fixed transmitter stations. A TOA measurement is made by tracking a specific zero-crossing of the received pulses (phase tracking) and resolving the cycle ambiguity by reference to the shape of the pulse envelope (cycle selection). Unfortunately, unwanted signals propagated as skywaves and superimposed on the wanted groundwave signal can reduce the accuracy of TOA measurements. The conventional technique for minimising such skywave interference is to measure the TOAs at a zero-crossing point that precedes the arrival of the earliest skywave component. However, skywave delays vary significantly with the time of day and season of the year. 
If the zero-crossing point chosen is earlier than it needs be in the conditions obtaining, the receiver will suffer unnecessarily low signal-to-noise and signal-to-interference ratios; if it is set too late, the receiver will suffer excessive skywave errors.

A new concept of receiver design, proposed by the authors and Bian $[1,2]$, continuously adaptively adjusts the sampling point to the optimal value in a constantly changing skywave interference environment. In the original proposal, a Fourier technique was used to identify the onset of the earliest skywave component, the TOA then being measured at the immediately preceding zero crossing. This Fourier approach, however, has rather limited time resolution. This letter presents an alternative, higher resolution, estimation technique using the autoregressive moving average (ARMA) algorithm. The performance of this new technique is evaluated by simulation and using off-air data under noisy conditions and compared with previous Fourier-based method.

\section{Signal model}

The Loran-C signal received, $x_{c}(t)$, may be represented in the time domain by [1]:

$x_{c}(t)=x_{g}(t)+\sum_{n=1}^{N} k_{n} x_{g}\left(t-\tau_{n}\right)+e(t)$

where $x_{g}(t)$ is the groundwave signal. The amplitude and delay of the $n t h$ skywave component relative to the groundwave, are represented by $k_{n}$ and $\tau_{n}$, respectively. The term $e(t)$ is the total noise and interference.

By taking the Fourier Transform (FFT) of equation (1), we obtain the equivalent representation of the composite signal in the frequency domain: 
$X_{c}(f)=X_{g}(f)\left[1+\sum_{n=1}^{N} k_{n} \exp \left(j 2 \pi f \tau_{n}\right)\right]+E(t)$

where $X_{c}(f), X_{g}(f)$ and $E(f)$ are the Fourier Transforms of $x_{c}(t), x_{g}(t)$ and $e(t)$, respectively.

\section{FFT Spectral-division Technique}

In the Fourier method we start by dividing the spectrum of the signal by the spectrum of a standard Loran-C pulse: we call this a "spectral-division" approach. We then return to the time domain by taking the Inverse Fourier Transform (IFFT) of the result. In the time domain we observe impulses at the arrival times of the groundwave and skywave components. This process is described by the following equation:

$$
F^{-1}\left[\frac{X_{c}(f)}{X_{0}(f)}\right]=k_{g}\left[\delta(t)+\sum_{n=1}^{N} k_{n} \delta\left(t-\tau_{n}\right)\right]+F^{-1}\left[\frac{E(f)}{X_{0}(f)}\right]
$$

where $F^{-1}$ is the Inverse Fourier Transform operator, $X_{0}(f)$ is the spectrum of the normalised standard Loran-C pulse $x_{0}(t)$ and $k_{g}$ is a constant related to the amplitude of the groundwave.

\section{The ARMA Technique}

This FFT spectral-division technique will be shown below to have limited resolution in time. The novel, higher-resolution, approach proposed employs an ARMA algorithm in place of the IFFT operation in equation (3). In the ARMA model, the input sequence $\varepsilon(n)$ (which is a white noise process with zero mean and variance $\sigma^{2}$ ), and the output sequence $x(n)$, are related by the following linear difference equation:

$x(n)=-\sum_{k=1}^{p} a_{k} x(n-k)+\sum_{k=0}^{q} b_{k} \varepsilon(n-k)$ 
where the $a_{k}{ }^{\prime} s$ and the $b_{k}{ }^{\prime} s$ are the ARMA model coefficients and $(p, q)$ is the order of the model.

The ARMA spectrum is determined by performing the following steps [3, 4]:

(1) Estimate the order required for the model by using the Akaike information criterion (AIC):

$$
\operatorname{AIC}(p, q)=-\ln \sigma^{2}+2\left(\frac{p+q}{N}\right)
$$

where $N$ is the number of samples.

(2) Determine the coefficients of the model by using the ARMA Yule-Walker normal equations:

$$
r_{x}(m)=\left\{\begin{array}{ll}
-\sum_{k=1}^{p} a_{k} r_{x}(m-k)+\sigma^{2} \sum_{k=m}^{q} b_{k} h(k-m) & 0 \leq m \leq q \\
-\sum_{k=1}^{p} a_{k} r_{x}(m-k) & m \geq q+1
\end{array}\right\}
$$

where $r_{x}(k)$ is the autocorrelation estimate, defined by:

$r_{x}(k)=\frac{1}{N} \sum_{n=0}^{N-1-k} x(n) x^{*}(n+k)$

(3) Compute the spectrum of the ARMA model from the following equation:

$$
P_{A R M A}(f)=\sigma^{2}\left|1+\sum_{k=1}^{q} b_{k} \exp (-j 2 \pi f k)\right|^{2} /\left|1+\sum_{k=1}^{p} a_{k} \exp (-j 2 \pi f k)\right|^{2} .
$$

The peaks of $P_{A R M A}(f)$ represent the frequencies of the composite signal. When estimating the delays of the skywave components of a Loran-C signal, the ARMA method is applied in the frequency domain. 


\section{Evaluation of performance}

A skywave delay estimation simulation has been developed to evaluate and compare the Fourier spectral division and ARMA methods [1,2]. Fig. 1 shows typical results when a groundwave component at $100 \mu s$ is followed by a skywave component $50 \mu s$ later and $12 \mathrm{~dB}$ stronger. The limited resolution of the FFT spectral-division method in Fig. 1a may be compared with the much higher resolution of the ARMA method in Fig. 1b. The sharper peaks of the new method allow the times of arrival of the groundwave and skywave components to be estimated much more accurately. These results were obtained at a signal-to-noise ratio at the receiver input of $-13 \mathrm{~dB}$, a performance $3 \mathrm{~dB}$ better than is required of Loran receivers by the US Coast Guard.

The ARMA technique has also been evaluated using off-air data. Fig. 2a shows a Loran pulse sequence received at Delft (Netherlands) from Sylt (Germany). ARMA analysis (Fig. 2b) shows the pulse to consist of a groundwave component starting at $55 \mu \mathrm{s}$ followed by a skywave component at $155 \mu \mathrm{s}$. The TOA detector can, therefore, be set at any time into the pulse up to this skywave delay of $100 \mu \mathrm{s}$. This experiment was the first time Loran-C skywave delays had been estimated using an ARMA algorithm. The FFT spectral-division analysis, in contrast, failed to resolve the arrival times for these off-air signals. It is believed that this was because of the greater sensitivity of the old method to distortion of the Loran-C pulse acquired as the signal propagated over land.

\section{Conclusions}

A novel signal processing technique using the ARMA algorithm for estimating skywave delays in Loran-C receivers has been presented. This technique has been shown to out- 
perform the FFT spectral-division algorithm in terms of resolution. This Letter has also demonstrated the superiority of the new technique using off-air data.

\section{Acknowledgement}

The authors thank Van Nee and other colleagues at Delft University, Netherlands, for the use of their off-air data.

\section{References}

1. Bian, Y. and Last, J.D., 'Novel Techniques for the Identification of Loran-C Skywaves', Proceeding of the 21st Annual Technical Symposium, Wild Goose Association, 1992, Birmingham, England, pp. 239-246.

2. Mohammed, A.F. and Last, J.D., 'Loran-C Skywave Delay Detection Using Rational Modelling Techniques', IEE Radio Receivers and Associate System Conference, 1995, Bath University, England, pp. 100-104.

3. Kaveh, M., 'High Resolution Spectral Estimation for Noisy Signals', IEEE Transaction on Acoustics, Speech and Signal Processing, 1979, 27 , (3), pp. 286-287.

4. Candy, J.V., 'Signal Processing: The Modern Approach', 1988, McGraw-Hill Inc., NJ, USA. 

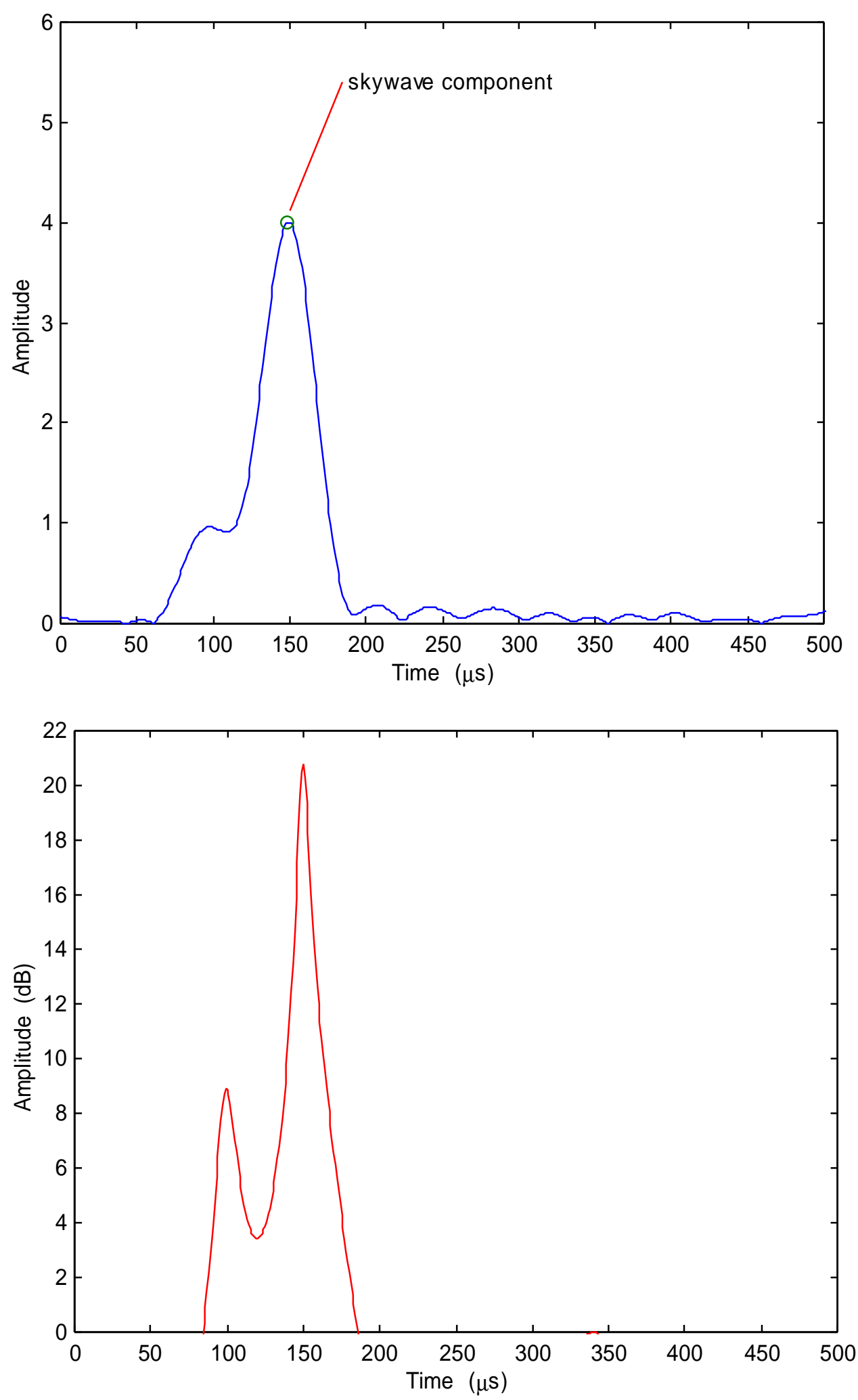

Fig. (1) 

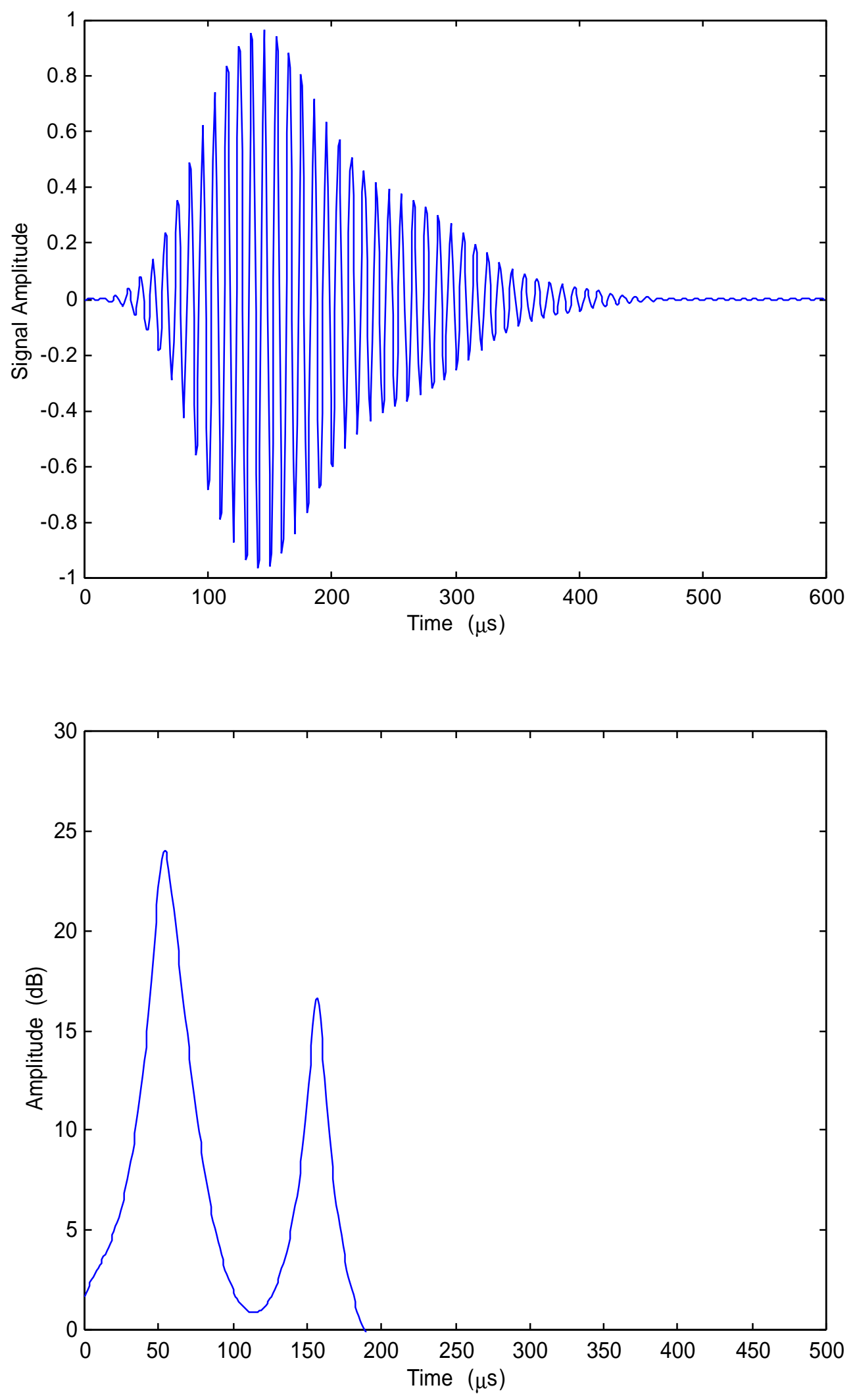

Fig. (2) 


\section{Figure Captions}

Fig. 1 Use of the two methods to analyse a Loran-C pulse consisting of a groundwave component followed by skywave component $50 \mu \mathrm{s}$ later and $12 \mathrm{~dB}$ stronger,

(a) FFT spectral-division method,

(b) ARMA algorithm.

Fig. 2 Test of the ARMA method using an off-air Loran-C signal,

(a) Off-air Loran-C pulse,

(b) Estimated arrival times. 